\title{
Spacecraft Swarm Navigation and Control Using Artificial Potential Field and Sliding Mode Control
}

Chakravarthini M. Saaj ${ }^{1}$, Vaios Lappas ${ }^{1}$ and Veysel Gazi ${ }^{2}$

${ }^{1}$ Surrey Space Centre, University of Surrey, UK, ${ }^{2}$ Department of Electrical and Electronics Engineering, TOBB University of Economics and Technology, Turkey.

c.saaj@surrey.ac.uk, v.lappas@surrey.ac.uk,v.gazi@etu.edu.tr

\begin{abstract}
The artificial potential field (APF) method provides simple and effective path planners for practical terrestrial robotics control. Sliding mode control (SMC) strategy together with artificial potential field has been used for control of multiagent systems or swarms. The aim of this work is to examine for the first time the applicability of APF and SMC for spacecraft swarm navigation and control. This paper demonstrates that spacecraft formation flying can be successfully achieved using SMC for closed loop feedback and APF method for path planning.
\end{abstract}

\section{INTRODUCTION}

The formation concept, first explored in the 1980's to allow multiple geostationary satellites to share a common orbital slot [1], has recently entered the era of application with many successful real missions (a few examples are: EO-1 in formation with LandSat-7, TechSat21). Swarms of microsatellites are currently envisioned as an attractive alternative to traditional large spacecraft. The main advantage of spacecraft formation flying is that by successfully controlling a formation, one may have them cooperate in a way that enables missions that would have required an enormous single spacecraft. The ability to distribute the functionality of one large spacecraft among several much smaller may reduce the total weight and in turn the launch costs. The failure of a single spacecraft in a formation does not necessarily lead to system failure as it would in a single larger spacecraft. Upgrades or repairs could be performed by simply replacing any obsolete or disabled spacecraft [2]. One of the most challenging problems with spacecraft swarming, formation flying and station keeping is that the spacecrafts must be autonomous and able to generate and correct their own relative positions with limited guidance from the ground. The first work in this field, as in the relative position case, stems from the work done on automatic rendezvous and docking control of two spacecraft, as was done on the Apollo missions. Later it was utilized in the Space Shuttle, Skylab and Gemini [3]. Ref. [4] developed a general optimization based control methodology to solve constrained trajectory generation problems for station keeping and reconfiguration of fully actuated low thrust micro-satellites. Ref. [5] designed an off-board computed procedure using the theory of optimal control for the design of formation reconfiguration. Although these results are encouraging, much work is needed before this technology is feasible for actual space applications.
A well-known approach to collision-free path planning of terrestrial robots is using Artificial Potential Field (APF) developed by [6]. APF is rapidly gaining popularity in many practical applications, as it is computationally less expensive than the global approach and provides a simple and effective path planner for obstacle avoidance. However, the major inherent drawback is the existence of local minima that differ from the desired configuration. Ref. [7] has proposed a method to avoid local minima using simulated annealing for local and global path planning of mobile robots. For space-related applications, the swarm of satellites will fail to attain the final target configuration if it is trapped in an undesirable local minimum. Variable structure control with a sliding mode was first described by Soviet authors including [8] and [9]. Today SMC is used as a general design method and is being examined for a wide spectrum of systems including nonlinear systems, multi-input/multi-output systems, discrete-time models, large scale and infinite-dimensional systems and stochastic systems. Numerous theoretical advances and practical applications have been reported in literature ([10], [11], [12], [13], [14]) to list a few. The methodology of using artificial potential field and sliding mode control for swarm aggregation and formation can be found in [15]. The APF method has a wide range of space applications, like path-constrained proximity maneuvers, largeangle attitude slew maneuvers, autonomous rendezvous and docking, self assembly and on-orbit servicing, and spacecraft formation and station keeping. An APF based approach for autonomous satellite navigation was proposed by [16] and selfassembly in space was proposed by [17].

This paper aims to further explore the applicability of SMC and APF algorithm presented in [15] for spacecraft swarm navigation and control. It is demonstrated that with APF method complex path planning can be achieved using minimum computational effort. Also the use of SMC makes the approach robust to a wide class of disturbances acting on the spacecraft due to interaction with the plasma environment. This paper is organized as follows: In Section II, the autonomous path planning technology using APF method developed in [15] is repeated. Section III describes the navigation and control strategy using APF method and SMC. The simulation results presented in Section IV discuss how the above algorithm can be effectively extended for spacecraft swarm navigation and control. The concluding remarks are drawn in Section V. 
II. Review of Path Planning using Artificial Potential FIELD

Some of the results in [15] relevant to this study are summarized here. Let the swarm consists of $N$ individual members or agents in the $n$ dimensional Euclidean space. The position of $i^{\text {th }}$ agent is described by $x^{i} \in R^{n}$. It is assumed that synchronous motion exists and there is no time delay. The motion of each agent in the swarm is governed by the equation [15]

$$
\dot{x^{i}}=\sum_{j=1, j \neq i}^{N} g\left(x^{i}-x^{j}\right), i=1, \ldots, N
$$

where, $g($.$) is an odd function which represents the sum of$ the function of attraction and repulsion between the agents. The function $g($.$) can be represented by$

$$
g(y)=-y\left[g_{a}(\|y\|)-g_{r}(\|y\|)\right]
$$

where $y \in R^{n}$ is arbitrary and $\|y\|=\operatorname{sqrt}\left(y^{T} y\right)$ is the Euclidean norm. Equation (1) can be represented also by

$$
\dot{x}^{i}=-\nabla_{i} J(x), i=1, \ldots, N
$$

where, $J: R^{n N} \rightarrow R$ is a potential function that represents the inter-individual interactions. Note that here the potential function $J(x)$ is not static. It depends on the relative positions of the individuals in the swarm. Therefore, uncertainties and disturbances including those acting on the system dynamics can affect the time derivative of $J(x)$. Consider potential function of the form

$$
J(x)=\sum_{i=1}^{N-1} \sum_{j=i+1}^{N} J_{i j}\left(\left\|x^{i}-x^{j}\right\|\right)
$$

where, $J_{i j}\left(\left\|x^{i}-x^{j}\right\|\right)$ is the potential between $i$ and $j$ and can be different for different pairs. Moreover, it is assumed that $J_{i j}\left(\left\|x^{i}-x^{j}\right\|\right)$ satisfies the following conditions:

A1) The potentials $J_{i j}\left(\left\|x^{i}-x^{j}\right\|\right)$ are symmetric and satisfy

$$
\nabla_{x^{i}} J_{i j}\left(\left\|x^{i}-x^{j}\right\|\right)=-\nabla_{x^{i}} J_{i j}\left(\left\|x^{i}-x^{j}\right\|\right)
$$

A2) There exist corresponding functions $J_{a}^{i j}: R^{+} \rightarrow R^{+}$ and $J_{r}^{i j}: R^{+} \rightarrow R^{+}$such that $J_{i j}()=.J_{a}^{i j}()-.J_{r}^{i j}($. and $\nabla_{y} J_{a}(\|y\|)=y g_{a}^{i j}(\|y\|)$ and $\nabla_{y} J_{r}(\|y\|)=y g_{r}^{i j}(\|y\|)$ where, $J_{a}^{i j}(\|y\|)$ the attractive potential function dominates on long distances and $J_{r}^{i j}(\|y\|)$ the repulsive potential function that dominates on short distance. Then, the above assumption restricts the motion of the individuals toward each other along the gradient of these potentials (i.e. along the combined gradient field of these potentials).

A3) There exists unique distance $\delta_{i j}$, at which for $g_{a r}^{i j}()=.g_{a}^{i j}()-.g_{r}^{i j}($.$) \quad we have g_{a r}^{i j}\left(\delta_{i j}\right)=0$ and $g_{a r}^{i j}(\|y\|)>0$ for $\|y\|>\delta_{i j}$ and $g_{a r}^{i j}(\|y\|)<0$ for $\|y\|<\delta_{i j}$.
Here $\delta_{i j}$ is the unique distance at which the attraction and the repulsion balance i.e., $g_{a}^{i j}\left(\delta_{i j}\right)=g_{r}^{i j}\left(\delta_{i j}\right)$. Functions satisfying the above conditions $\mathrm{A} 1-\mathrm{A} 3$ result in aggregating swarm behavior. Assuming that the motion of the individuals is given by (1) it can be shown that the following hold.

R1) The center $\bar{x}=N^{-1} \sum_{i=1}^{N} x^{i}$ of the swarm is stationary for all time.

R2) If $J(x)$ is bounded from below. i.e., $J(x)>a$ for some finite $a \in R$, then, for any initial condition $x(0) \in R^{n N}$, as $t \rightarrow \infty$ we have $x(t) \rightarrow \Omega_{e}$ where $\Omega_{e}=\{x: x=0\}$ and $x^{T}=\left\lfloor\begin{array}{lll}x^{1 T} & \ldots & x^{N T}\end{array}\right]$.

R3) The swarm size will be bounded, and the position $x^{l}$ of all the agents will converge asymptotically to a small region around its center $\bar{x}$, which is a hyperball of size $\varepsilon, B_{\varepsilon}(\bar{x})=\{y:\|y-\bar{x}\| \leq \varepsilon\}$. Moreover, the convergence to $B_{\varepsilon}$ will occur in a finite time.

It is inferred that given the initial positions of the individuals $x^{i}(0), i=1, \ldots, N$, the final configuration to which the individuals will converge is unique. However, it is difficult to find a direct relation between $x(0)$ and the final position $x(\infty)$. This is a shortcoming of this approach. Now under the stated assumptions on the potential function the equation of motion in (1) with the pair dependent attraction/repulsion becomes

$$
x^{i}=\sum_{j=1, j \neq i}^{N} g_{i j}\left(x^{i}-x^{j}\right), i=1, \ldots, N
$$

Where the attraction/repulsion function $g_{i j}($.$) for all pairs$ $(i, j)$ satisfy $g_{i j}\left(x^{i}-x^{j}\right)=-g_{j i}\left(x^{j}-x^{i}\right)$. For formation control the attraction and repulsion functions and therefore the equilibrium distance $\delta_{i j}$ for different pairs of individuals can be different. The desired formation can be uniquely specified with respect to rotation and translation by the formation constraints $\left\|x^{i}-x^{j}\right\|=d_{i j}$ for all $(i, j), j \neq i$. The idea is to chose each of the attraction/repulsion functions $g_{i j}($.$) such$ that $\delta_{i j}=d_{i j}$ for every pair of individuals $(i, j)$. The generalized Lyapunov function $J(x)=\sum_{i=1}^{N-1} \sum_{j=i+1}^{N}\left[J_{a}^{i j}\left(\left\|x^{i}-x^{j}\right\|\right)-J_{r}^{i j}\left(\left\|x^{i}-x^{j}\right\|\right)\right]$

then have its minimum at the desired formation and once the formation is achieved $x^{i}=0$ for all $i$. If $J(x)$ is chosen as a formation function, which has a unique minimum at the 
desired formation, then the desired formation will be asymptotically achieved.

III. Spacecraft NAVIGATION AND CONTROL USING Artificial Potential Field And SLiding Mode Control

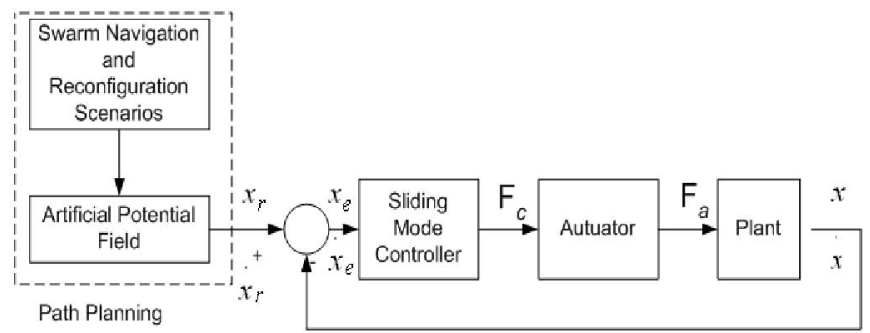

Fig. 1. Schematic diagram of path planning and control

The schematic diagram of the proposed navigation and control architecture is shown in Figure 1. It integrates various sensory signals to achieve collision-free goal oriented navigation and formation reconfiguration. The path-planning module is capable of avoiding obstacles and provides a goal-oriented navigation in an optimal time period. In artificial potential field method, the dynamic environment in which the spacecraft exists is represented by a scalar potential function, which has a minimum potential (sink) at the desired terminal state of the vehicle dynamics and has maximum potential (source) at path constraints (near-by satellites or obstacles). In other words, the potential of the spacecraft swarm will be constructed by identifying each satellite in the swarm as a region of high potential. A repulsive force between them, which is simply the negative gradient of the potential field, avoids collisions between the neighboring spacecrafts. Since the rate of descent of the potential function is rendered negative definite, the potential-field approach guarantees that the spacecraft will converge to the desired terminal state without violating the defined path constraints. The convergence time depends on dissipative terms (damping) in the control law. The final formation will be achieved only if every member of the swarm is in a sink corresponding to the target configuration. This approach has less computational load as compared to deliberative techniques that carry out extensive map building from raw sensory data. Fig. 2 illustrates the force experienced by a satellite in a swarm of three satellites using APF method. It is desired that the Swarm Agent 2 (SA2) move towards the final target position. Initially, SA1 exerts a repulsive force, and the target exerts an attractive force on SA2. Then it moves to a new location in the direction of the resultant force. At this position, SA3 exerts a repulsive force and target exerts an attractive force on SA2 and it moves towards the next intermittent location. Here the target exerts the attractive force and SA2 finally moves towards its target position, thereby achieving the desired configuration. In this approach the steering direction of a particular spacecraft undergoing reconfiguration within the spacecraft swarm is determined by assuming that the other members of the constellation (obstacles) assert repulsive forces on the spacecraft and the goal (desired terminal state) asserts attractive force. Consequently, the spacecraft experiences a generalized force equal to the negative of the total potential gradient that drives the spacecraft towards the goal or the desired terminal state. In this way the APF provides a constantly active navigation, offering a collision free trajectory for the each of the individual

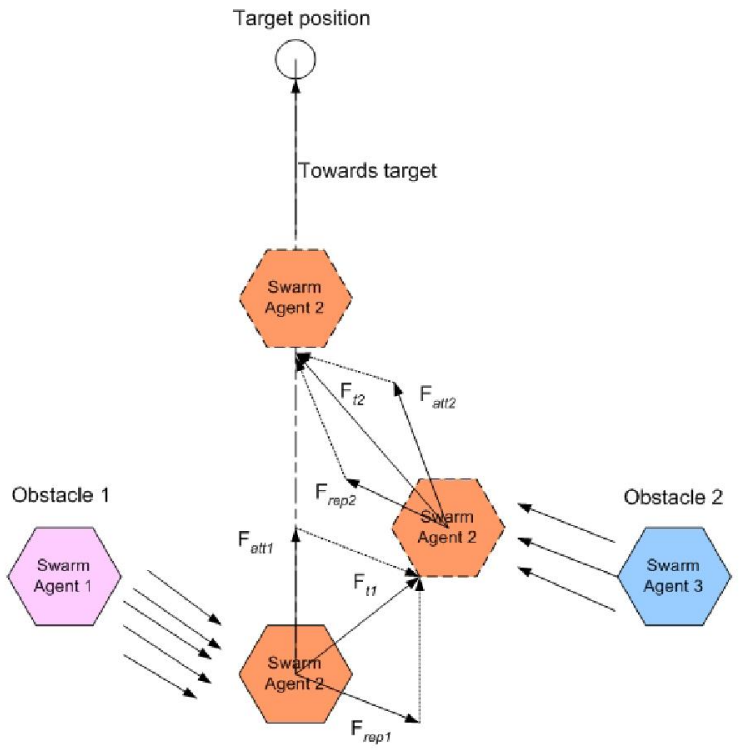

Fig. 2. Schematic diagram of path planning using APF

satellites that form part of a constellation or formation. Sliding mode control is a powerful robust nonlinear control technique that enables separation of the overall system motion into independent partial components of lower dimensions, and as a result reducing the complexity of the control design. The aim is to design each of the control inputs such that a cohesive swarming is achieved thereby preserving the results in the previous section. To ensure this, we need to enforce satisfaction of Equation (2). In short, the control inputs are designed such that the velocity of the agents are enforced along the negative gradient of the potential function $J(x)$. Consider the nonlinear inertial equation of motion of the spacecraft

$$
M_{i}\left(x^{i}\right) \ddot{x^{i}}+f_{i}\left(x^{i}, \dot{x^{i}}\right)=u^{i}, 1 \leq i \leq N
$$

where $x^{i} \in R^{n}$ is the position vector of agent $i, M_{i} \in R^{n \times n}$ is the mass or inertia matrix, $N$ is the number of agents in the swarm, $f_{i}\left(x^{i}, x^{i}\right) \in R^{n}$ represents the centripetal, Corriolis, gravitational effects and additive disturbances, and $u^{i} \in R^{n}$ represents the control inputs. It is assumed that $M_{i}$ is nonsingular and $\underline{M}_{i} \leq M_{i} \leq \bar{M}_{i}$ where $\underline{M}_{i}>0$ and $\bar{M}_{i}$ are the known lower and upper bounds of the inertia matrix respectively. For all $i$ the matrix $M_{i}\left(x^{i}\right)$ satisfies

$$
\underline{M}_{i}\|y\|^{2} \leq y^{T} M_{i}\left(x^{i}\right) y \leq \bar{M}_{i}\|y\|^{2}
$$


Let

$$
f_{i}\left(x^{i}, x^{i}\right)=f_{i}^{k}\left(x^{i}, x^{i}\right)+f_{i}^{u}\left(x^{i}, x^{i}\right), 1 \leq i \leq N
$$

where $f_{i}^{k}(.,$.$) represents the known part and f_{i}^{u}(.,$.

represents the unknown part and $\bar{f}_{i}$ are known constants for all $i$ such that $\left\|f_{i}^{u}\left(x^{i}, x^{i}\right)\right\| \leq \bar{f}_{i}, 1 \leq i \leq N$. In classical sliding mode control problems, the surface $s=0$ is chosen such that on it, the tracking error asymptotically decays to zero. The design of the sliding mode surface considered here is a little different from conventional approach. In their approach, the surfaces $s^{i}=0$ are chosen so that the system motion obeys certain dynamics. The major difference is that the sliding surfaces $s^{i}$ 's are not constant surfaces and they can move as the agents move even though $\dot{x}^{i}$ can be viewed as the output of the system and $s^{i}$ as the output error and at $s^{i}=0$ the output errors becomes zero.

The $n$-dimensional sliding manifold for agent $i$ is

$$
s^{i}=\dot{x}^{i}+\nabla_{x^{i}} J(x)=0, i=1, \ldots, N
$$

Once all the agents reach their sliding manifolds $s^{i}=0$, (5) reduces to $x^{i}=-\nabla_{i} J(x)$ which is same as the motion equation (2) of the agents in the swarm. A sufficient condition for sliding mode to occur is given by [9]

$$
s^{i T} s^{i}<0, \forall i=1, \ldots N
$$

This guarantees that starting from any initial point in the state space the sliding manifold is reached asymptotically. Differentiating (5) we obtain

$$
\dot{s^{i}}=\ddot{x}^{i}+\frac{\partial}{\partial t}\left(\underset{x^{i}}{.} J(x)\right)
$$

From (4) we get

$$
\ddot{x^{i}}=M_{i}^{-1}\left(x^{i}\right)\left[u^{i}-f_{i}\left(x^{i}, \dot{x^{i}}\right)\right]
$$

Substituting (8) in (7) and then in (6) we get the necessary conditions for occurrence of sliding mode as

$$
s^{i T}\left[M_{i}^{-1}\left(x^{i}\right) u^{i}-M_{i}^{-1}\left(x^{i}\right) f_{i}\left(x^{i}, \dot{x^{i}}\right)+\frac{\partial}{\partial t}\left(\nabla_{x^{i}} J(x)\right)\right]<0 .
$$

If the potential function $J(x)$ chosen such that $\nabla_{y} J(x)$ is bounded i.e., $\left\|\frac{\partial}{\partial t}\left(\nabla_{y} J(x)\right)\right\| \leq \bar{J}$ for some known bound $\bar{J}$ and for all $y \in R^{n}$, then the control input $u^{i}$ will enforce the sliding mode condition. The sliding mode controller that enforce the occurrence of sliding mode takes the form

$$
u^{i}=-u_{o}^{i}(x) \operatorname{sign}\left(s^{i}\right)+f_{i}^{k}\left(x^{i}, x^{i}\right)
$$

where, $\operatorname{sign}\left(s^{i}\right)=\left[\begin{array}{lll}\operatorname{sign}\left(s_{1}^{i}\right) & \ldots & \operatorname{sign}\left(s_{N}^{i}\right)\end{array}\right]^{T}$. The gain of control input is chosen as

$$
u_{o}^{i}>{\underline{M_{i}}}^{-1}\left(\overline{M_{i}} \bar{f}_{i}+\bar{J}+\varepsilon^{i}\right),
$$

for some $\varepsilon^{i}>0$. This guarantees occurrence of sliding mode in finite time. In the above controller only the known part $f_{i}^{k}\left(x^{i}, \dot{x}^{i}\right)$ of the vehicle dynamics is utilized. If there are no known parts, then the controller would reduce to $u^{i}=-u_{o}^{i}(x) \operatorname{sign}\left(s^{i}\right)$. Also note that the controller design does not need the exact mass/inertia matrix $M_{i}\left(x^{i}\right)$ of the spacecraft. It only needs the upper and lower bounds on them as is the case also with disturbances. Consider the Lyapunov function of the form

$$
V_{i}=0.5 s^{i T} s^{i} .
$$

Differentiating (10) with respect to time gives $\dot{V}_{i} \leq-\varepsilon^{i} \operatorname{sqrt}\left(V_{i}\right)$ and this guarantees that the sliding surface is reached in a finite time bounded by $t_{\max }^{i}=2 V_{i}(0) / \varepsilon^{i}$ and sliding mode occurs on all the surfaces $s^{i}=0$ in a finite time bounded by $\bar{t}_{S m}=\max _{i=1, \ldots, N}\left(2 V_{i}(0) / \varepsilon^{i}\right)$. In order to reduce the chattering phenomenon, the $\operatorname{sign}\left(s^{i}\right)$ term in the controller can by replaced by $\tanh \left(\beta s^{i}\right)$. Due to the chattering problem in practical implementations, it may not be possible to ideally recover all the stability results in previous section. For example the statement (R1) that the center of the swarm is stationary for all time may not necessarily hold and there might be small deviations of the center. Nevertheless, it is expected that most of the results (such as swarm cohesiveness and finite time convergence) can be obtained will only small perturbations.

\section{Simulation Study}

This section illustrates the results of the simulation studies performed for the tetrahedron formation using sliding mode control and artificial potentials discussed in the preceding section. The specifications used for the simulation are listed in Table 1

TABLE I

\section{List of Simulation Parameters}

\begin{tabular}{|l|l|}
\hline Simulation parameter & Value/Units \\
\hline Spacecraft individual mass $M$ & $150 \mathrm{~kg}$ \\
\hline Lower bound on mass $\underline{M}$ & $0.5 \mathrm{M}$ \\
\hline Upper bound on mass $\bar{M}$ & $1.5 M$ \\
\hline Spacecraft radius $\eta$ (assumed spherical) & $0.5 \mathrm{~m}$ \\
\hline Number of s/c for tetrahedron formation $N$ & 4 \\
\hline Final inter spacecraft formation separation $d$ & $50 \mathrm{~m}$ \\
\hline
\end{tabular}


For simulation purpose we used $N=4, n=3, \varepsilon_{i}=1$, $\bar{J}=20 N, \beta=10$ and $\bar{f}_{i}=500 N$. The uncertainty acting in the system is $f_{i}\left(x^{i}, x^{i}\right)=500 \sin (0.2 t)$. Note that the use of SMC permits the use of large upper bounds on the disturbance acting on the spacecrafts due to plasma interactions. For formation control each agent in the swarm is pre-assigned a desired position in the final formation. The potential function considered for formation control problem that satisfies the assumptions in Section II is the function with linear attraction and exponential repulsion terms is given by

$$
g(y)=-y\left(a-b \exp \left(-\left\|y^{2}\right\| / c\right)\right.
$$

where, $a, b$ and $c$ are positive constants such that $b>a$. The constant $a$ is in a sense the magnitude of the attraction and $b$ is in a sense the magnitude of the repulsion and the constant $c$ is its spread or repulsion range but the actual repulsion is some combination from effects of both. Let $a=b \exp \left(-d^{2} / c\right)$, $b=0.015, c=2500$ and $d=50$. For formation control each agent in the swarm is pre-assigned a desired position in the final formation. The parameter $a$ is computed in order to achieve the balance of attraction and repulsion between any two agents at the desired distance $d$ in the final tetrahedron formation. By increasing the repulsive force (i.e. by increasing $b$ ) we could guarantee collision avoidance. By equating (11) to zero, it can be seen that $g(y)$ switches sign at the set of points defined by $\Psi=\{y=0$ or $\|y\|=\delta=\operatorname{sqrt}(c / \ln (b / a))\}$. The convergence to $B_{\varepsilon}$ will occur in a finite time bounded by $\bar{t}=\max _{i=1, \ldots, N}\left\{-(2 a)^{-1} \ln \left(\varepsilon^{2} /\left\|x^{i}(0)-\bar{x}(0)\right\|^{2}\right\}\right.$. From the plot of this potential function in Fig. 3, we see that the attraction range the repulsion range and the point it crosses the horizontal axis is the desired formation separation $(d=50 \mathrm{~m})$. The distance at which attraction balance the repulsion is at a unique distance $\delta=\operatorname{sqrt}(c / \ln (b / a))=50$. Fig. 4a shows the trajectories of the spacecrafts initialized at rest with random initial positions. With time the four spacecrafts move to their required inter spacecraft separation of $50 \mathrm{~m}$ and form the required tetrahedron formation while avoiding collisions. Fig. $4 \mathrm{~b}$ shows the final

formation positions and the center of the formation $x$ is represented by ' $*$ '. During transient before the occurrence of

sliding mode it is possible for the center $\bar{x}$ to move. However, once the sliding mode occurs the center is expected to be almost stationary. For practical implementations, there might be small deviations even after transient, which are due to chattering effects. Fig. 5a shows the minimum, average and the maximum distances between the individuals in the formation. It is seen that the average distance between spacecrafts in the tetrahedron formation is equal to the side length of the tetrahedron or the final inter- spacecraft separation of $50 \mathrm{~m}$. Fig. $5 \mathrm{~b}$ shows the minimum, average and maximum of the distances of the individual spacecraft positions to the formation center. As expected, the average distance to the center $\bar{x}$ is equal to $50 \operatorname{sqrt}(3 / 8)$.

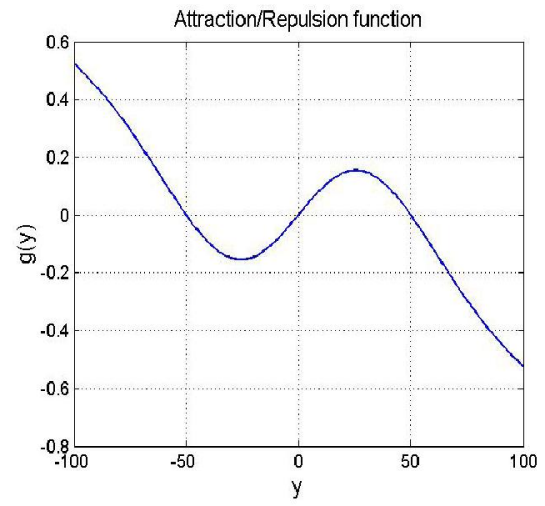

Fig.3. Attraction/repulsion function.

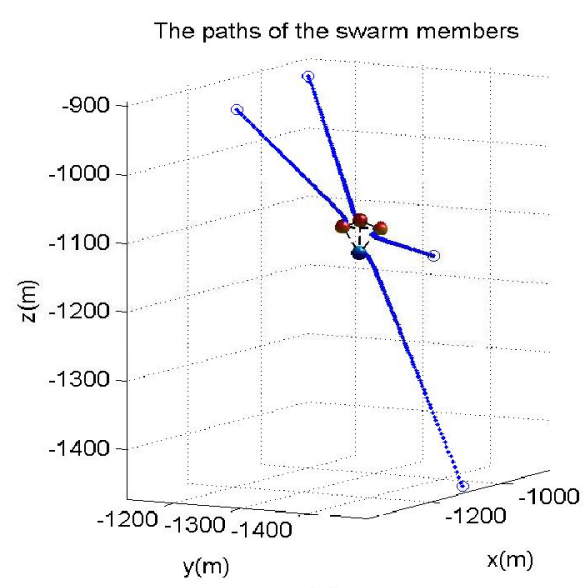

(a)

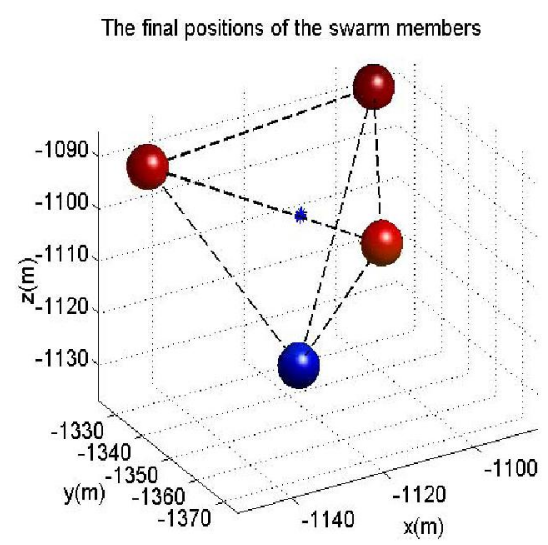

(b)

Fig.4. Simulation results: Tetrahedron formation (a)-(b) 


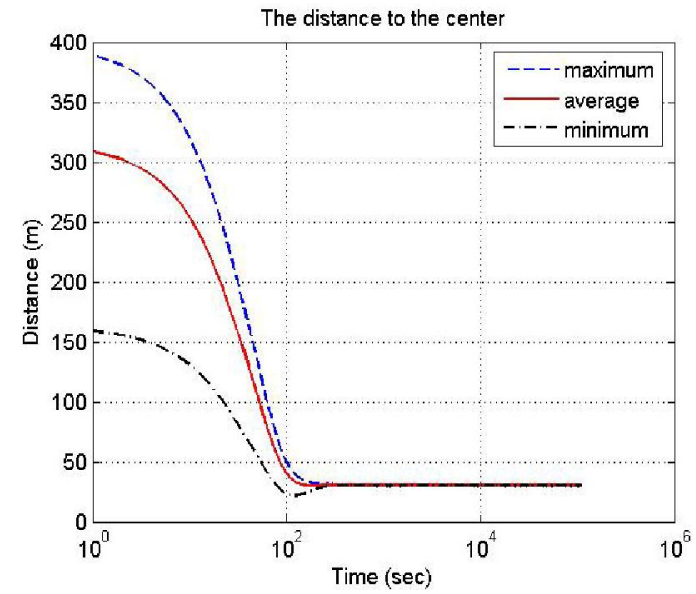

(a)

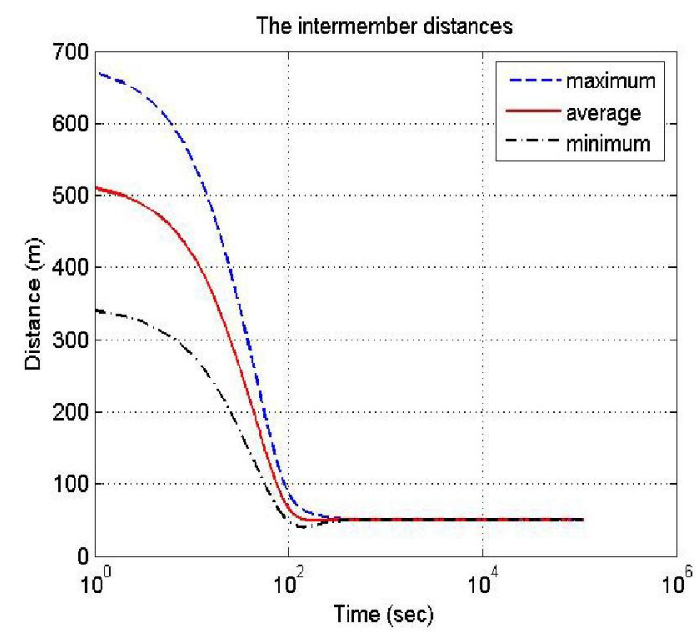

(b)

Fig. 5. Simulation results: Tetrahedron formation (a)-(b)

\section{CONCLUSIONS}

We studied the feasibility of an algorithm for autonomous path planning and navigation of swarm of satellites using artificial potential field method combined with sliding mode control. It is expected that this method will ensure safe and autonomous navigation and reconfiguration of the spacecrafts in the formation. We examined the tetrahedron formation in sufficient technical detail to evaluate the merits and demerits associated with their eventual implementation. The major conclusions drawn from this study are the following:

- The autonomous navigation and reconfiguration using artificial potential field is suitable for both spacecraft swarm formation flying.

- The major advantage of using sliding mode controller is the inherent insensitivity to parameter variations and disturbances once in the sliding mode, thereby eliminating the necessity of exact modeling. However, proper chatter elimination technique should be used to make this suitable for practical missions.

The feasibility of this approach for various other scenarios like spacecraft swarm aggregation and hexagon formation has been successfully studied in parallel and the simulation studies using actuator dynamics are under progress.

\section{ACKNOWLEDGEMENTS}

The results in this paper are part of the project supported by the European Space Agency Advanced Concept Team under the Ariadna programme. This support is gratefully acknowledged.

\section{REFERENCES}

[1] J. G. Walker, "The Geometry of Cluster Orbits", J. Brit. Interplan. Soc., vol. 35 , pp. $345,1982$.

[2] A. Das, R. Cobb and M. Stallard, "TechSat 21 - A Revolutionary Concept in Distributed Space Based Sensing," Proc. of the Guidance, Navigation and Control Conference, vol. AIAA-98-5255, Boston, MA, pp. 1-6, 1998.

[3] P. Wang, F. Hadaegh. and M. Mokuno, "Formation flying of multiple spacecraft with automatic rendezvous and docking capability", AIAA Guidance, Navigation and Control Conference and Exhibit Proceedings, 2003.

[4] M. B. Milam, N. Petit and R. M. Murray, "Constrained Trajectory Generation of Micro-Satellite Formation Flying", AIAA Guidance Navigation and Control Conference, 4030, 2001.

[5] M. Campbell, "Planning Algorithm for Multiple Satellite Clusters", $J$. Guidance Control and Dynamics, vol. 26, no. 5, pp. 770-780, 2003.

[6] O. Khatib, "Real-Time Obstacle Avoidance for Manipulator and Mobile Robots", The Int. J Robotics Research, vol. 5, no. 1, pp. 90-98, 1986.

[7] M. G. Park, J. H. Jeon and M. C. Lee, "Obstacle Avoidance for Mobile Robots using Artificial Potential Field Approach with Simulated Annealing", IEEE Int. Symposium on Industrial Electronics, Pusan, Korea, pp. 1530-1535, 2001.

[8] S. V. Emel'yanov, "Design of variable structure control systems with discontinuous switching functions," Eng. Cybern., vol. 1, pp. 156-160, 1964.

[9] V. I. Utkin, "Equations of the slipping regime in discontinuous systems: I," Autom. Remote Control, vol. 32, pp. 1897-1907, 1971.

[10] B. S. Heck and A. A. Ferri, "Application of output feedback to variable structure systems," Journal of Guidance, vol. 12, pp. 932-935, 1989.

[11] J. Y. Hung, W. Gao and J. C. Hung, "Variable structure control: A survey", IEEE Trans. Industrial Electronics, vol. 40, no. 1, pp. 2-22, 1993.

[12] K. D. Young, V. I. Utkin and U. Ozguner, "A control engineer's guide to sliding mode control", IEEE Trans. Control System, vol. 7, no. 3, pp. 328-342, 1999.

[13] C. M. Saaj, B. Bandyopadhyay and H. Unbehauen, "A new algorithm for discrete-time sliding mode control using fast output sampling feedback", IEEE Trans. on Industrial Electronics, vol. 49, pp. 518-523, 2002.

[14] S. Janardhanan and B. Bandyopadhyay, "Discrete sliding mode control of systems with unmatched uncertainty using multirate output feedback", IEEE Trans. Automatic control, vol. 51, no. 6, pp. 1030-1035, June 2006.

[15] V. Gazi, "Swarm aggregations using artificial potentials and sliding mode control", IEEE Transactions on Robotics, vol. 21, no. 6, pp. 1208-1214, 2005.

[16] C. R. McInnes, "Potential Function Methods For Autonomous Spacecraft and Control", Paper AAS 95-447, 1995.

[17] F. McQuade, "Autonomous Control for On-Orbit Assembly Using Artificial Potential Functions", Ph.D. thesis, Faculty of Engineering University of Glasgow, 1997. 